\section{Deregulation required?}

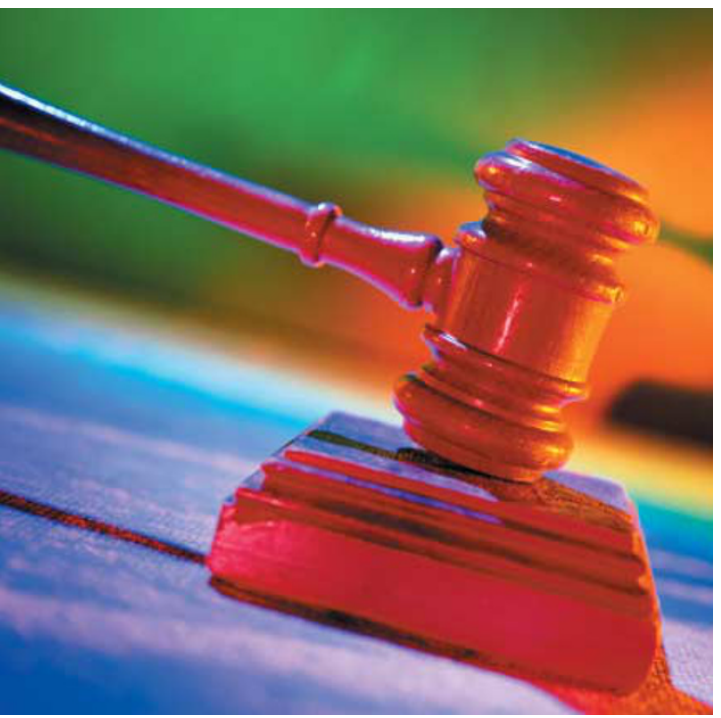

Patients with cancer have detectable numbers of $\mathrm{T}$ cells in their blood that recognize tumour-associated antigens, yet this rarely corresponds with effective tumour eradication, even after tumour-vaccine-induced increases in cytotoxic T-cell numbers. Curiel and colleagues now show that regulatory $\mathrm{T}$ cells might be one factor that prevents an effective antitumour response.

Recent studies have indicated that when selfrecognizing $\mathrm{T}$ cells are present, regulatory $\mathrm{T}$ cells become activated and shut down the antitumour response. Curiel and colleagues studied the profile of tumour-associated T cells in patients with advanced ovarian cancer and found that $10-17 \%$ of $\mathrm{T}$ cells isolated from the tumour ascites were regulatory $\mathrm{T}$ cells $\left(\mathrm{CD} 4{ }^{+} \mathrm{CD} 25^{+}\right)$. Furthermore, about $23 \%$ of the $\mathrm{T}$ cells identified in the tumour tissue itself were of the regulatory subtype, with the percentage of these cells increasing as disease progressed. However, regulatory $\mathrm{T}$ cells were undetectable in normal ovarian tissue sections.

But what effect do regulatory $\mathrm{T}$ cells have on the antitumour immune response? The authors showed that regulatory $\mathrm{T}$ cells isolated from human tumour ascites inhibited the proliferation of autologous tumour-specific cytotoxic T cells in a mouse xenograft model of ovarian cancer. And although injection of tumourspecific cytotoxic T cells delayed tumour growth, this effect was inhibited by the co-injection of regulatory $\mathrm{T}$ cells.

What might attract a regulatory $\mathrm{T}$ cell into the tumour tissue? Regulatory T cells are normally localized in lymph nodes, but surprisingly, there were fewer regulatory $\mathrm{T}$ cells in tumour-draining nodes than in lymph nodes from patients without cancer. Indeed, with increasing cancer progression, the levels of regulatory $\mathrm{T}$ cells decreased in the lymph nodes and increased within the tumour tissue and ascites, indicating that regulatory $\mathrm{T}$ cells might be actively recruited from these sites by

\section{TUMORIGENESIS}

\section{Two faces of hypoxia}

Hypoxia is often thought of as something cancer cells need to overcome to survive, necessitating the formation of new blood vessels to escape from areas of low oxygen. However, a growing body of evidence indicates that hypoxia might actually promote cancer development. Adding to this evidence, a recent study from Eileen White and colleagues shows that hypoxia drives cancer progression by promoting genomic instability, and that inactivation of apoptosis is essential for tumour-cell survival during this process.

The authors were interested in the role of the pro-apoptotic BCL2-family proteins BAK and $\mathrm{BAX}$ in tumorigenesis. In baby mouse kidney epithelial cells transformed with the adenoviral $E 1 A$ oncogene and a dominantnegative form of TP53, apoptosis is partially blocked - because of p53 inactivation - but the $\mathrm{p} 53$-independent apoptotic pathway that involves BAX and BAK is still functional. These cells are transformed in vitro, but do not form tumours when injected into animals, raising the question of why inactivation of the BAX/BAK apoptotic pathway is required for tumorigenesis.

White and colleagues investigated whether this is because these cells that express functional $\mathrm{BAK}$ and $\mathrm{BAX}$ are unable to survive in the hypoxic conditions that tumour cells encounter in vivo. Consistent with this, they found that the injected cells were subjected to high levels of hypoxia, resulting in cell death by apoptosis and necrosis. By contrast, despite being exposed to hypoxic conditions in vivo, cells in which the BAX/BAK pathway had been inactivated - either by deletion of Bax and Bak, or by overexpression of the anti-apoptotic protein BCL2 - survived to form tumours.

Interestingly, cells lacking BAX and BAK function formed giant polyploid tumour cells in vivo, whereas cells with functional BAX and BAK did not. This led White and colleagues to suggest that hypoxia promotes polyploidy, but that blocking the p53independent apoptotic pathway is required for cells to survive this process.

To directly test whether inactivation of the BAX/BAK pathway is required for survival in hypoxic conditions, the authors used an in vitro ischaemia assay, in which cells are deprived of both oxygen and nutrients. Transformed cells expressing E1A and dominant-negative $\mathrm{p} 53$, but with functional $\mathrm{BAK}$ and $\mathrm{BAX}$, underwent apoptosis in these conditions, whereas cells in which the BAK/BAX pathway was inactivated survived.

To test if this promoted genomic instability, the authors subjected cells to ischaemia, followed by a period of recovery, and looked at chromosome content and levels of apoptosis. Ischaemic conditions resulted in the generation of cells with increased chromosome content, regardless of the status of the BAX/BAK pathway. However, for cells in which this pathway was active, high levels of apoptosis were seen, and no polyploid cells were observed after the recovery period. By contrast, inactivation of BAX and BAK function led to the survival and persistance of polyploid cells.

Polyploidy is thought to be an important step in generating the genomic instability and aneuploidy that have been linked to tumour progression. So, it seems that by promoting this process, hypoxic and ischaemic conditions have a key role in cancer progression, but that loss or blockcade of the BAX and BAK pathway is required for survival during this step. This provides a new insight into the molecular changes that are needed for tumours to evolve, and might have important implications for therapeutic strategies that aim to kill cancer cells by inducing hypoxia.

\section{Louisa Flintoft}

(D) References and links

ORIGINAL RESEARCH PAPER Nelson, D. A. et al. Hypoxia and defective apoptosis drive genomic instability and tumorigenesis. Genes Dev. 18, 2095-2107 (2004).

FURTHER READING Harris, A. L. Hypoxia - a key regulatory factor in tumour growth. Nature Rev. Cancer 2 38-47 (2002)

WEB SITE

Eileen White's lab:

http://white.cabm.rutgers.edu/index.html 